\title{
INTERNAL COMPLETENESS AND INJECTIVITY OF BOOLEAN ALGEBRAS IN THE TOPOS OF $M$-SETS
}

\author{
M. Mehdi Ebrahimi
}

In this paper we study internal completeness, injectivity and some related notions in the category MBoo of Boolean algebras in the topos MEns of $M$-sets, for a monoid $M$.

In Section 1, we deal with the notion of internal completeness in MBoo and show that an algebra $A$ in $\mathbf{M B o o}$ is internally complete if and only if the embedding [.]: $A \longmapsto N(A)$ of $A$ into the algebra $N(A)$ of (internal) normal ideals of $A$ is an isomorphism.

In Section 2, we study the notion of injectivity and essential extensions in MBoo and show that: injectivity implies internal completeness; the injective hull of 2 is $H(2)$, the algebra of all subsets of $M$, if an only if $M$ is a finite group; for a finite monoid $M, 2$ is injective if and only if $M$ has a right absorbing element; and for a finite and commutative monoid $M$, a subalgebra $A$ of $H(2)$ is an essential estenstion of 2 if and only if $A$ is generated by the blocks of a monoid congruence $\theta$ on $M$ with $M / \theta$ being a group. Further, we give examples to show that the latter result is not true in general. MBoo.

Finally, in Section 3, we characterise the subdirectly irreducible algebras in

\section{Preliminaries}

0.1 For a monoid $M$ let MEns be the topos of all (left) $M$-sets (sets with a left $M$-action) and the equivariant maps between them. Considering $M$ as a category with one object, MEns is the functor category Ens $^{\mathbf{M}}$ where Ens is the category of sets. Hence, the subobject classifier $\Omega$ of this topos is the set of all left ideals of $M$ (subsets of $M$ which are closed under the left multiplication) together with the action of $M$ on $\Omega$ given by division, that is for $t \in M$ and $S \in \Omega, t S=\{s \in M \mid s t \in S\}$. The

Received 30 May 1989

This work was done during my sabbatical leave spent at McMaster University. I wish to express my deep appreciation to B. Banaschewski for valuable discussions, suggestions and encouragement throughout my work. I must say that without his help and generous assistance I could not have completed this work.

Financial assistance from the Natural Science and Engineering Research Council of Canada, through their operating grant to B. Banaschewski, is gratefully acknowledged. Also, I would like to thank the University of Shahid Beheshti (The National University of Iran) for granting me this sabbatical leave.

Copyright Clearance Centre, Inc. Serial-fee code: 0004-9729/90 \$A2.00+0.00. 
true map $1 \longrightarrow \Omega$, where $1=\{0\}$ is the terminal object of MEns, takes 0 to $M$, the largest ideal of $M$. For any subobject $A \stackrel{r}{\longrightarrow} B$, the classifying map $f_{\tau}: B \longrightarrow \Omega$ is defined by $f_{\tau}(b)=\{x \in M \mid x b \in A\}$. Hence $f_{\tau}(b)=M$ if and only if $b \in A$. Notice that $\Omega=\{\phi, M\}$ if and only if $M$ is a group. For $A \in$ MEns, a global element $f: 1 \longrightarrow A$ is given by an element $f(0)$ of $A$ which is fixed under the action of $M$. Since $t S=M$ if and only if $t \in S$, one can check easily that $\Omega$ has exactly two global elements. This shows that the topos MEns is bivalued. But MEns is a Boolean topos if and only if $M$ is a group.

0.2 The power set $\mathcal{P}(M)$ of $M$ is an $M$-set with the action given by division, and $\Omega$ is a pseudo-complemented subalgebra of $\mathcal{P}(M)$. The pseudo-complement of $S \in \Omega$ is

$$
S^{*}=\{s \in M \mid(\forall t \in M)(t s \notin S)\}
$$

It is easily checked that $\Omega$ is a Stone algebra, that is $S^{*} \cup S^{* *}=M$, if and only if $S^{*}=\emptyset$ for all $S \neq \emptyset$ in $\Omega$. Also, by [9], $\Omega$ is a Stone algebra if and only if $M$ satisfies the (left) Ore condition, that is, for any $a, b$ in $M$, there exist $s, t$ in $M$ such that $s a=t a$. In fact, if the Ore condition is satisfied, $S \neq \emptyset$ is in $\Omega$ and $a \in S$, then for any $b \in M$, there exist $s, t$ in $M$ with $t b=s a$. Now, since $S$ is a left ideal of $M$, $t b=s a \in S$. This gives us that $b \notin S^{*}$, and hence $S^{*}=\emptyset$.

0.3 For $A, B$ in MEns, $B^{A}$ is the set of all equivariant maps $f: M \times A \longrightarrow B$, together with the action of $M$ defined by

$$
(s f)(t, a)=f(t s, a)
$$

for $s, t$ in $M$ and $a \in A$. It is easily sseen that $\Omega^{A} \cong \operatorname{Sub}(M \times A)$ subobjects of $M \times A$. For any subobject $X$ of $M \times A$, we have

$$
X=\bigcup_{\in \in M}\{s\} \times X,
$$

where $X,=\{a \in A \mid(s, a) \in X\}$. Hence we can identify $X$ by a family $\left(X_{0}\right)_{s \in M}$, where for each $s \in M, X$, is a subset of $A$ with

$$
(\forall t \in M)\left(a \in X, \Rightarrow t a \in X_{t s}\right) .
$$

The action of $M$ on $\Omega^{A}$ is then given by

$$
t X=\left(X_{a t}\right)_{s \in M} .
$$

0.4 In the following, MLatt will denote the category of lattices in MEns, with lattice maps preserving the $M$ action, and $\mathrm{MBoo}$ is the category of Boolean algebras in MEns. For any algebra $A$, the underlying object of $A$ is denoted by the same letter A. 


\section{Internal Completeness}

1.1 For $A \in$ MLatt, $\operatorname{Id}(A)$ and $\operatorname{MId}(A)$ denote the set of ideals of (the lattice) and $M$-ideals of (the $M$-lattice) $A$, respectively. The action of $M$ on $\operatorname{Id}(A)$ is given by $s \cdot J=[s J]$, for $s \in M$ and $J \in \operatorname{Id}(A)$, where $[s J]$ is the ideal of $A$ generated by the set $s J=\{s x \mid x \in J\}$. That is

$$
s \cdot J=\{a \in A \mid(\exists x \in J)(a \leqslant s x)\} .
$$

The action of $M$ on MId $(A)$ is defined in the same way.

It is clear that the map $\downarrow: A \rightarrow \operatorname{Id}(A)$ defined by a $\mapsto \downarrow a=\{x \in A \mid x \leqslant a\}$ is an equivariant map.

1.2 For $A \in \mathbf{M B o o , ~ t h e ~ i n t e r n a l ~ i d e a l ~ l a t t i c e ~} \mathcal{J}(A)$ of $A$ in the topos MEns is given by

$$
\mathcal{J}(A)=\left\{X=\left(X_{s}\right)_{s \in M} \mid\left(X \in \Omega^{A}\right) \&(\forall s \in M)(X, \in \operatorname{Id}(A))\right\}
$$

For $X=\left(X_{s}\right)_{s \in M}$ and $Y=\left(Y_{s}\right)_{s \in M}$, their meet and join in $\mathcal{J}(A)$ is defined component-wise, that is

$$
X \wedge Y=\left(X_{s} \wedge Y_{s}\right)_{s \in M} \text { and } X \vee Y=\left(X_{s} \vee Y_{s}\right)_{s \in M}
$$

where $X_{\varepsilon} \wedge Y_{2}$ is their intersection and $X_{2} \vee Y_{s}$ is their join in $\operatorname{Id}(A)$, that is $X, \vee Y_{s}=$ $\left\{b \vee c \mid b \in X_{s}, c \in Y_{s}\right\}$. The action of $M$ on $\mathcal{J}(A)$ is the same as the action on $\Omega^{A}$. Notice that if $M$ is a group, then $\mathcal{J}(A)=\operatorname{Id}(A)$.

One also defines a lattice embedding $[\cdot]: A \longmapsto \mathcal{J}(A)$ by $[a]=(\downarrow s a)_{s \in M}$, which preserves the action of $M$.

In addition, one defines a lattice embedding ()$^{\#}: \operatorname{Id}(A) \longmapsto \mathcal{J}(A)$, preserving the action, by $J^{\#}=([s J])_{, \in M}$, for $J \in \operatorname{Id}(A)$. Since $(\downarrow a)^{\#}=[a]$, the following diagram is commutative

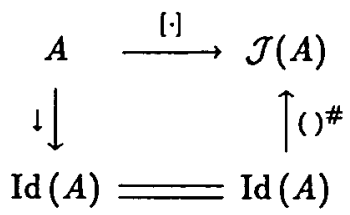

1.3 Recall that a lattice $A$ in MEns in internally complete (see [8], p.147) if there exists an order preserving equivariant map $V: \mathcal{J}(A) \longrightarrow A$ which is (internally) left adjoint to $[\cdot]: A \longmapsto \mathcal{J}(A)$. That is, for $X=\left(X_{s}\right) s \in M$ in $\mathcal{J}(A)$ and $c \in A$,

$$
V X \leqslant c \Longleftrightarrow X \leqslant[c] \Longleftrightarrow(\forall s \in M)(X, \leqslant \downarrow s c) .
$$

By Proposition 5.35 and 5.36 in $[8], \Omega$ and $\Omega^{A}$ are internally complete. 
Proposition 1.4. If $A \in$ MBoo is internally complete, then $A$ is complete in Boo and the action of $\mathrm{M}$ on $A$ is complete (that is preserves the join).

Proof: Since the underlying functor $U: \mathrm{MBoo} \longrightarrow$ Boo has a left adjoint, which preserves pullbacks, $A$ is complete as a Boolean algebra (see Proposition 5.6) [8]). In fact the join map $\widetilde{V}: \operatorname{Id}(A) \longrightarrow A$ is given by the following commutative diagram

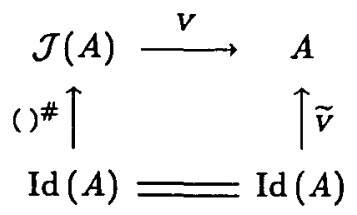

That is, $\widetilde{V} J=V J^{\#}$, for $J \in \operatorname{Id}(A)$. Since the maps ()$^{\#}$ and $V$ are both equivariant, so is $\widetilde{V}$.

The converse of the above proposition is not true in general. Consider the initial object 2 of MBoo. By [9], 2 is internally complete if an only if $\Omega$ is a stone algebra, that is if and only if $M$ satisfies the left Ore condition (see 0.2 ).

If $M$ is a group, then $A \in \mathrm{MBoo}$ is internally complete if and only if $A \in \mathbf{B o o}$ is complete. This is because $\mathcal{J}(A)=\operatorname{Id}(A)$, and the actions are isomorphism (onto) and hence complete, for the join in Boo is defined by means of the order and the actions preserve the order.

Let $A \in$ MBoo and $J \in \mathcal{J}(A)$. The (internal) pseudo-complement $J^{*}$ of $J$ is defined by

$$
\begin{aligned}
J_{s}^{*} & =\left\{x \in A \mid(\forall t \in M)\left(t x \in\left(J_{t s}\right)^{*}\right)\right\} \\
& =\bigcap_{t \in M} t^{-1}\left(J_{t s}\right)^{*}
\end{aligned}
$$

for each $s \in M$. We now prove that $J^{*}$ is indeed the pseudo-complement of $J$.

LEMMA 1.5. $J^{*}$ is the pseudo-complement of $J$.

Proof: $J^{*}$ is in $\mathcal{J}(A)$, because each $\left(J_{t s}\right)^{*}$ is in $\operatorname{Id}(A)$ and, since $t^{-1}\left(J_{t_{s}}\right)^{*}$ is in $\operatorname{Id}(A), J_{s}^{*}=\bigcap_{t \in M} t^{-1}\left(J_{t s}\right)^{*}$ belongs to $\operatorname{Id}(A)$. If $x \in J_{s}^{*}$ and $t_{0} \in M$, then for any $t \in M, t\left(t_{0} x\right)=\left(t t_{0}\right) x$ belongs to $\left(J_{t t_{0} s}\right)^{*}$, and hence $t_{0} x \in J_{t_{0}}^{*}$. Now, $x \in J_{\mathbf{a}} \cap J_{s}^{*}$ implies that $x \in J_{0}$ and $x \in J_{a}^{*}$. Hence $t x \in\left(J_{t a}\right)^{*}$, for all $t \in M$, which implies that $x \in\left(J_{s}\right)^{*}$. Hence, by the definition of $\left(J_{a}\right)^{*}, x=x \wedge x=0$.

Now let $J \wedge H=0$, for some $H \in \mathcal{J}(A)$. Then $J_{s} \cap H_{s}=0$, for all $x \in M$. To show that $H_{s} \subseteq J_{s}^{*}$, let $h \in H_{s}$. To see that $t h \in\left(J_{t s}\right)^{*}$, for all $t \in M$, let $b \in J_{t s}$. Now $t h \wedge b \in J_{t s}$ and since $t h \in H_{t s}, t h \wedge b \in H_{t s}$. Then $t h \wedge b$ being in $J_{t s} \cap H_{t s}=0$ implies that $t h \in\left(J_{t s}\right)^{*}$. Hence $J^{*}$ is indeed the pseudo-complement of $J$.

Notice that, since $(t \cdot J)^{*}=t \cdot J^{*}$, the map ()$^{*}: \mathcal{J}(A) \longrightarrow \mathcal{J}(A)$ is equivariant. 
Lemma 1.6. If $A \in$ MBoo is internally complete and $J \in \mathcal{J}(A)$, then

$$
J^{*}=\left[(V J)^{\prime}\right]
$$

where ( $)^{\prime}$ is complementation in $A$.

Proof: Let $c=V J$. To see that for each $s \in M$,

$$
\mathcal{J}_{\varepsilon}^{*}=\left\{x \mid t x \in\left(J_{t s}\right)^{*}\right\}=\downarrow s c^{\prime},
$$

Let $x \in J_{a}^{*}$. Then we have

$$
\begin{aligned}
x \leqslant s c^{\prime} & \Longleftrightarrow s c \leqslant x^{\prime} \\
& \Longleftrightarrow s V J \leqslant x^{\prime} \\
& \Longleftrightarrow V s \cdot J \leqslant x^{\prime} \\
& \Longleftrightarrow s \cdot J \leqslant\left[x^{\prime}\right] \\
& \Longleftrightarrow(\forall t \in M)\left((s \cdot J)_{t} \leqslant \downarrow t x^{\prime}\right) \\
& \Longleftrightarrow(\forall t \in M)\left(J_{s t} \leqslant \downarrow t x^{\prime}\right) \\
& \Longleftrightarrow(\forall t \in M)\left(\left(\downarrow t x^{\prime}\right)^{*} \leqslant\left(J_{s t}\right)^{*}\right) \\
& \Longleftrightarrow(\forall t \in M)\left(\downarrow t x \leqslant\left(J_{s t}\right)^{*}\right) \\
& \Longleftrightarrow(\forall t \in M)\left(t x \in\left(J_{s t}\right)^{*}\right) \\
& \Longleftrightarrow\left(x \in J_{s}^{*}\right) .
\end{aligned}
$$

1.7 Let $N(A)=\left\{J \in \mathcal{J}(A) \mid J=J^{* *}\right\}=\left\{J^{*} \mid J \in \mathcal{J}(A)\right\}$. That is $N(A)$ is the equaliser of ()$^{* *}: \mathcal{J}(A) \longrightarrow \mathcal{J}(A)$ and the identity map $\mathcal{J}(A) \longrightarrow \mathcal{J}(A)$. We call this the algebra of (internal) normal ideals of $A$. Since $[\cdot]^{*}=\left[()^{\prime}\right]$, the embedding $[\cdot]: A \longmapsto \mathcal{J}(A)$ factors through $N(A)$. This shows that any algebra $A$ in MBoo can be embedded into an internally complete one. That $N(A)$ is internally complete follows from the fact that the usual proof is constructively valid.

The above lemma shows that

Proposition 1.8. A MBoo in internally complete if and only if $[\cdot]: A \longrightarrow$ $N(A)$ is an isomorphism.

\section{INJECTIVITY IN MBoo}

2.1 Recall that an object $A$ in a category is injective if and only if for any morphism $h: B \longrightarrow A$ and any monomorphism $g: B \longmapsto C$, there exists a morphism $f: C \longrightarrow A$ such that $f g=h$. Further, a monomorphism $h: A \longmapsto B$ is called essential if any 
$g: B \longrightarrow C$ for which $g h$ is a monomorphism is itself a monomorphism. In MBoo, one checks easily that $h: A \longmapsto B$ is essential if and only if every $M$-ideal of $B$ with zero inverse image by $h$ is itself zero. The result of Ebrahimi [4] and the classical facts about Boolean algebras show that for any Grothendieck topos, and hence in particular for MEns, every algebra $A \in$ MBoo has an injective hull (that is an essential injective extension). That is, the category MBoo has enough injectives.

2.2 Consider the following adjointness

$$
\text { MEns } \underset{H}{\stackrel{v}{\rightleftarrows}} \text { Ens }
$$

with the underlying functor $U$ a left adjoint of the functor $H$ defined by : for any $X \in \operatorname{Ens}, H(X)$ is the set of all functions from the set $M$ to the set $X$, with the action of $M$ on $H(X)$ given by $(s f)(t)=f(t s)$, for $f \in H(X)$ and $s, t \in M$. This adjointness can be lifted to

$$
\text { MBoo } \underset{H}{\stackrel{U}{\rightleftarrows}} \text { Boo }
$$

denoted by the same letters. Since $H$ has a left adjoint $U$ which preserves finite limits, and hence monomorphisms, $H$ preserves injective and complete Boolean algebras. In particular, for $2 \in \mathbf{B o o}$, the algebra $H(2)$ of all subsets of $M$ is injective and internally complete.

Lemma 2.3. [.]: $A \longmapsto N(A)$ is essential.

Proof: Let the composite $A \stackrel{[\cdot]}{\longrightarrow} N(A) \stackrel{\varphi}{\longrightarrow} B$ be a monomorphism; that is, for $x \in A, \varphi[x]=\varphi\left[(\downarrow s x)_{s \in M}\right]=0$ implies that $x=0$. Let $X=\left(X_{s}\right)_{s \in M}$ be in $N(A)$ and $\varphi(X)=0$. Let $X, \neq 0$, for some $s \in M$. Then $X,=(s \cdot X)_{e} \neq 0$, where $e$ is the identity of $M$. Let $x \in(s \cdot X)_{e}$, then, for any $t \in M, t x \in(s \cdot X)_{t e}$, and hence $\downarrow t x \subseteq(s \cdot X)_{t}$, for $(s \cdot X)_{t}$ in an ideal of $A$. Thus $[x] \leqslant s \cdot X$, and hence $\varphi[x] \leqslant \varphi(s \cdot X)=s \varphi(X)=0$. Thus $x=0$, and hence $X,=0$, which proves the lemma.

Proposition 2.4. If $A \in$ MBoo is injective, then it is internally complete.

Proof: By the above lemma, $N(A)$ is essential over $A$ and since $A$ is injective $A \cong N(A)$. Hence, by $1.8, A$ is internally complete.

The converse of the above proposition is not true in general: for a nontrivial (finite) group $M, 2$ is internally complete in MBoo but it is not injective, since $H(2)$ is an essential extension of 2 . In fact we have the following proposition which is a special case of Lemma 1.9 of [3].

Proposition 2.5. $H(2)$ is an essential extension of 2 if and only if $M$ is a finite group. 
Proof: It is clear that an algebra $E$ in MBoo is essential over 2 if and only if it is simple, that is $M \operatorname{Id}(E) \cong 2$. Let $M$ be a finite group, and $I \neq 0$ be an $M$-ideal of $H(2)$. Let $\emptyset \neq K \in I$, and $s \in K$. Since $I$ is an $M$-ideal, $s K=\{t \mid t s \in K\}$ in $I$, and hence $\{e\} \subseteq s K$ is in $I$. Now, for any $m \in M, m^{-1}\{e\}=\{m\}$ is in $I$. Since $M$ is finite, $M=\bigcup_{m \in M}\{m\}$ belongs to $I$. This shows that $H(2)$ is simple, and hence essential over 2. Conversely, let $H(2)$ be essential over 2 . Let $S \subseteq M$ be the set of all right invertible elements of $M$. For $K \subseteq S$ and $s \in M, s K=\{t \mid t s \in K\}$ is a subset of $S$. Hence the set of all subsets of $S$ is a (nontrivial) $M$-ideal of $H(2)$. By essentialness, we get that $M=S$. This shows that $M$ is a group. Now, since $M$ is a group, it is checked easily that the set $P_{f}(M)$ of all finite subsets of $M$ is a (nontrivial) $M$-ideal of $H(2)$. By essentialness, $M \in P_{f}(M)$ and hence $M$ is finite.

Proposition 2.6. The injective hull of 2 in MBoo is $H(2)$ if and only if $M$ is a finite group.

REMARK 2.7. For any monoid $M$, the injective hull of 2 in $M B o o$ is $H(2) / \mathcal{J}$ for a maximal $M$-ideal $J$ of $H(2)$.

Proposition 2.8. For a finite monoid $M, 2$ is injective in MBoo if and only if $M$ has a "right absorbing" element a (that is, as $=a$ for all $s \in M$ ).

Proof: Let 2 be injective. Since $H(2)$ is injective, there exists $h: H(2) \longrightarrow 2$. Now $h^{-1}\{1\}$ is an $M$-ultrafilter on $M($ in $H(2))$. Since $M$ is finite, $h^{-1}\{1\}$ is generated by $\{a\}$, for some $a \in M$, that is

$$
\bigcap_{\substack{h(X)=1 \\ X \subseteq M}} X=\{a\} .
$$

Now, for any $s \in M, h(s\{a\})=\operatorname{sh}(\{a\})=s \cdot 1=1$. This implies that $\{a\} \subseteq s\{a\}$. But $s\{a\}=\{x \in M \mid x s=a\}$, hence $a s=a$ for all $s \in M$. Conversely, let $a \in M$ be a right absorbing element. Consider $\mathcal{A}=\{X \mid a \in X \subseteq M\}=\uparrow\{a\}$ in $H(2)$. For any $s \in M, a \in s X$, because $s X=\{x \in M \mid x s \in X\}$ and $a s=a$ is in $X$. Hence, for $X \in \mathcal{A}$, as $=a \in X$ implies that $a \in s X$. Thus $\mathcal{A}$ is an $M$-ultrafilter on $M$. Thus, there exists $h: H(2) \longrightarrow 2$ given by

$$
h(X)= \begin{cases}1 & \text { if } a \in X \\ 0 & \text { if } a \notin X\end{cases}
$$

which is an MBoo morphism. Hence 2 retracts the injective algebra $H(2)$. This shows that 2 is injective.

Notice that the finiteness of $M$ is not needed to prove the converse of the above proposition. 
Proposition 2.9. Let $M$ be a finite and commutative monoid. Then a subalgebra $A$ of $H(2)$ is an essential extension of 2 if and only if $A$ is generated by (the blocks) of a monoid congruence $\theta$ on $M$ with $M / \theta$ being a group.

Proof: Since $M$ is commutative, the maps $\lambda_{t}: A \rightarrow A$ induced by the action of $t \in M$ are endomorphisms. Since $A$ is essential over 2 , and hence simple, the $\lambda_{t}$ 's are one to one. By finiteness of $A$, the $\lambda_{t}$ 's are isomorphisms. Now, for $\theta=\{(s, t) \mid$ $\left.\lambda_{\varepsilon}=\lambda_{t}\right\}, M / \theta$ is a group.

To see that $A$ is generated by the $\theta$-blocks, let $E \in A$ be the atom of $A$ containing $e$, which exists because $A$ is finite. If $\lambda_{1}=\operatorname{Id}=\lambda_{e}$, that is $s \in \theta[e]$, the $\theta$ block of $e$, then $s E=E$ and hence $s \in E$. This shows that $\theta[e]=E$. On the other hand, for $s \in E, E \subseteq s E$, because $e \in s E$ and $E$ is an atom. Since the $\lambda_{t}$ 's are automorphisms, $s E$ is an atom, and hence $E=s E$. Further $s(t E)=t E$, by commutativity, and thus $\lambda_{s}=\mathrm{Id}$, leaving all the atoms of $A$ fixed; every atom of $A$ is of the form $t E$ for some $t \in M$, because $M=t_{1} E \cup \ldots \cup t_{k} E$ for suitable $t_{1}, \ldots, t_{k}$.

Finally, if $\lambda_{\bar{s}}=\lambda_{s}^{-1}$, then $s E=\theta[\bar{s}]$, because $(x s, e) \in \theta$ if and only if $(x, \bar{s}) \in \theta$. This shows that $A$ is generated by the blocks of a congruence $\theta$ on $M$ with $M / \theta$ being a group.

Conversely if $\theta$ is a monoid congruence on $M$ with $M / \theta$ being a group, then $s \theta[e]=\theta[\bar{s}]$ for $s \bar{s}=e$ and the $\theta$-blocks generate a subalgebra $A$ of $H(2)$ which is an essential extension of 2 .

EXAMPLE 2.10. Consider the monoid $M=\{e, a, b\}$ with $x y=y$, for $x, y$ in $M$. The algebra

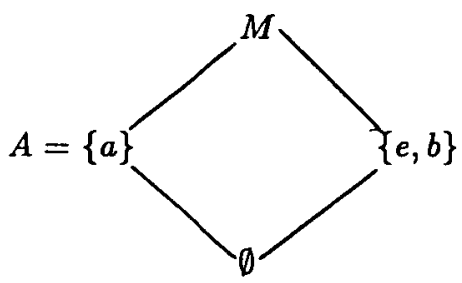

in MBoo is an essential extension (in fact the injective hull) of 2, but the partition $\{\{a\},\{e, b\}\}$ which generates $A$ is not a monoid congruence.

In fact, for the monoid $M=\left\{e, a_{1}, \ldots, a_{n}\right\}$ with $x y=y$, for $x, y$ in $M$, the algebra 


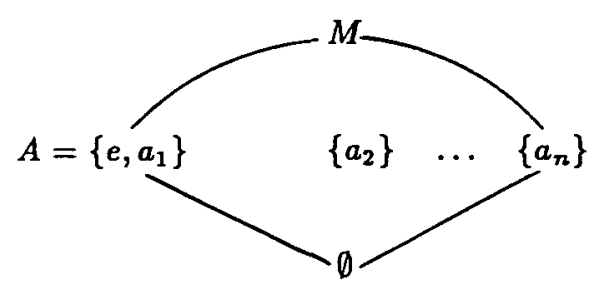

is the injective hull of 2.

\section{SUBDIRECT IRREDUCIBLITY}

Recall that an algebra $A$ is a category is subdirectly irreducible if and only if for any monomorphism $f: A \longmapsto \prod_{i \in I} A_{i}$, there exists an $i \in I$ with $p_{i} f: A \longrightarrow A_{i}$ a monomorphism, where $p_{i}$ is the $i$-th projection. For $A \in \mathbf{M B o o}$, this is equivalent to $A$ having a smallest nonzero $M$-ideal. For a different proof of the following see [7].

Lemma 3.1. If $A \in$ MBoo is subdirectly irreducible, then $A$ can be embedded into $H(2)$.

Proof: For $A \in$ MBoo, $U(A)$ is in Boo (see 2.2) and by the Representation Theorem in Boo, there exists a set $S$ with a monomorphism $U(A) \longrightarrow 2^{S}$. Hence we have

$$
A \longmapsto H U(A) \longmapsto H\left(2^{S}\right) \simeq(H 2)^{S} .
$$

Now, since $A$ is subdirectly irreducible, $A \longmapsto H(2)$ is an embedding.

Lemma 3.2. Every subalgebra $A$ of $H(2)$ is subdirectly irreducible.

Proof: Suppose $A$ is not subdirectly irreducible. Thus there exists a family $I_{\lambda}(\lambda \in \Lambda)$ of nontrivial $M$-ideals of $A$ whose intersection is trivial. Since the $I_{\lambda}$ 's are nontrivial, there are nonempty sets $X_{\lambda}$ in $I_{\lambda}$, for each $\lambda$. For each $\lambda$, take $s_{\lambda} \in$ $X_{\lambda}$. Since the $I_{\lambda}$ 's are $M$-ideals, $s_{\lambda} X_{\lambda} \in I_{\lambda}$, hence the intersection $X$ of the sets $s_{\lambda} X_{\lambda}(\lambda \in \Lambda)$ belongs to the intersection of the $M$-ideals $I_{\lambda}(\lambda \in \Lambda)$ which is trivial. Now, $X=\left\{x \in M \mid(\forall \lambda)\left(x s_{\lambda} \in X_{\lambda}\right)\right\}$, and hence $e \in X$, that is $X \neq \emptyset$ which is a contradiction.

By the last two lemmas we get

PROPOSITION 3.3. An algebra in MBoo is subdirectly irreducible if and only if it is isomorphic to a subalgebra of the algebra $H(2)$.

\section{REFERENCES}

[1] R. Balbes and P. Dwinger, Distributive Lattices (University of Missouri Press, 1975). 
[2] B. Banaschewski and K.R. Bhutani, 'Boolean algebras in a localic topos', Math. Proc. Cambridge Philos. Soc. 100 (1986), 43-55.

[3] S. Burris and M. Valeriote, 'Expanding varieties by monoids of endomorphisms', Algebra Universalis 17 (1983), 150-169.

[4] M.M. Ebrahimi, 'Algebra in a Grothendieck topos: injectivity in quasi-equational classes.', J. Pure. Appl. Algebra 26 (1982), 269-280.

[5] R. Goldblatt, Topoi: The Categorical Analysis of Logic (North-Holland, Amsterdam, 1979).

[6] P. Halmos, Lectures on Boolean Algebras (van Nostrand, Princeton, 1963).

[7] J. Ježek, 'Subdirectly irreducible and simple Boolean algebras with endomorphisms', in Universal Algebra and Lattice Theory: Lecture Notes in Math. 1149, Proc., Charleston, 1984, pp. 150-162 (Springer-Verlag, Berlin, Heidelberg, New York, 1985).

[8] P.T. Johnston, Topos Theory (Academic Press, 1977).

[9] P.T. Johnstone, 'Conditions related to de Morgan's law': Lectuure Notes in Math. 753, Proc., Durham 1977, pp. 479-491 (Springer-Verlag, Berlin, heidelberg, New York).

[10] S. MacLane, Categories for the Working Mathematician (Graduate Texts in Mathematics, No 5, Springer-Verlag, Berlin, Heidelberg, New York, 1971).

[11] R. Sikorski, Boolean Algebras (Ergebnisse der Math. Band 25 Springer-Verlag, Berlin, Heidelberg, New York, 1964).

Department of Mathematics

University of Shahid Beheshti

Tehran

Iran 